\title{
LA FORMACIÓN DE PALABRAS POR COMPOSICIÓN DESDE UN PUNTO DE VISTA HISTÓRICO
}

JUAN SÁNCHEZ MÉNDEZ

Université de Neuchâtel (Suiza)

Desde un punto de vista diacrónico no existe todavía un estudio completo y monográfico sobre la conformación y evolución histórica de la formación de palabras por composición en español. Carecemos de una historia de este proceso en español, lo que no es el caso ni para los estudios que ofrecen una descripción y clasificación sincrónicas o modernas de los compuestos, ni para los varios y extensos estudios históricos sobre la derivación mediante prefijos y sufijos. De hecho, salvo las referencias a la diacronía de determinados compuestos en los estudios sobre la formación de palabras, o salvo los trabajos dispersos dedicados al análisis histórico de determinados tipos compositivos, apenas encontramos nada que aborde históricamente el proceso en conjunto.

Un ejemplo claro de lo que estamos diciendo lo observamos al consultar algunas gramáticas históricas. Una característica común, que se repite estudio tras estudio, es el pequeño apartado de unas pocas páginas que se le brinda a la composición, al contrario de lo que ocurre con el procedimiento de la derivación, a la que se le dedica una detallada extensión mucho mayor con abundantes y pormenorizados datos. Además, no se ofrece tampoco una delimitación precisa entre derivación y composición, lo que sería necesario por cuanto históricamente los límites entre una y otra pueden resultar borrosos. De esta manera, podríamos considerar el espacio que las gramáticas históricas dan a los compuestos como un apéndice, algo impreciso, a la formación de palabras y su historia.

Dentro de lo que es la composición propiamente dicha, en manuales, como el de Hanssen ${ }^{1}$, Menéndez Pidal ${ }^{2}$, García de Diego ${ }^{3}$, entre otros, o en los más

\footnotetext{
${ }^{1}$ Federico Hanssen, Gramática histórica de la lengua castellana, Buenos Aires, El Ateneo, 1913.

${ }^{2}$ Ramón Menéndez Pidal, Manual de gramática histórica española, 13ª ed., Madrid, EspasaCalpe, 1968.

${ }^{3}$ Vicente García de Diego, Gramática histórica española, $3^{\mathrm{a}}$ ed., Madrid, Gredos, 1970.
} 
modernos como el de Ralph Penny ${ }^{4}$, tras definir el proceso de composición y de palabra compuesta, se procede seguidamente a clasificar, en primer lugar, los distintos tipos de compuestos en virtud de la naturaleza, de la estructura y de la formación de la composición (sin unanimidad entre los distintos autores, por lo que la clasificación puede variar significativamente) y, en segundo lugar, dentro de cada grupo de compuestos, según el carácter funcional de los constituyentes que se ponen en relación. En todos los casos se plantea el estudio desde la perspectiva interna del sistema, únicamente como un proceso morfológico, haciendo abstracción de consideraciones externas de la historia de la lengua que podrían arrojar luz sobre determinados compuestos y su desarrollo. Es decir, no plantean la posibilidad de considerar el compuesto también como una palabra o lexía surgida para responder a determinadas necesidades comunicativas o expresivas. A esto se añade, como venimos diciendo, que el apartado dedicado a la composición se centra más en explicar determinados compuestos particulares que en ofrecer una visión de conjunto del proceso de composición y de su evolución histórica en tanto que tal proceso, limitándose tan sólo a dar unas pocas y vagas ideas que apuntan hacia hechos generales.

Asimismo, la forma en que son tratados los compuestos, especialmente cuando se la compara con la derivación, pone de manifiesto la carencia de una base teórica del análisis histórico de la composición. Queremos decir que en la mayor parte de las gramáticas históricas el escaso espacio dedicado a la composición podría inscribirse perfectamente en un estudio sincrónico o incluso descriptivo de este mecanismo en español moderno si no fuera por las escuetas y algo escasas menciones a la diacronía o historia de algunos tipos compositivos y la presencia de voces antiguas, ya en desuso.

Por otra parte, salvo el enjundioso tratado sobre la composición nominal en español de Bustos Gisbert ${ }^{5}$, o el pormenorizado y extenso capítulo dedicado a ésta de J. F. Val ${ }^{6}$, en el que podemos ver la aplicación al español de determinados modelos teóricos y concepciones, los estudios sobre la formación de palabras por composición han sido escasos en nuestro ámbito. De un cotejo de la mayoría de los trabajos sobre la formación de palabras en español de las últimas décadas ${ }^{7}$ se infiere que apenas hay avances en metodología de análisis de

\footnotetext{
${ }^{4}$ Ralph Penny, Gramática histórica del español, $2^{\mathrm{a}}$ ed., Barcelona, Ariel, 2006.

${ }^{5}$ Eugenio de Bustos Gisbert, La composición nominal en español, Salamanca, Ediciones de la Universidad de Salamanca, 1986.

6 José Francisco Val, «La composición», en Ignacio Bosque y Violeta de Demonte, dirs. Gramática descriptiva de la lengua española, Madrid, Espasa, Vol III, 1999, págs. 4.757-4.842.

7 Véanse, por ejemplo, los trabajos de J. Alemany, Tratado de la formación de palabras en la lengua castellana, Madrid, Suárez, 1920, Hernán Urrutia, Lengua y discurso en la creación léxica. La lexicogenesia, Cupsa, Madrid, 1978, M. F. Lang, Spanish word formation, London, Routledge, 1990, José Alberto Miranda, La formación de palabras en español, Salamanca, Ediciones Colegio de España, 1994, Manuel Alvar Ezquerra, La formación de palabras en español,
} 
la composición, y tan sólo difieren en algunos aspectos concretos de la descripción, como los de la clasificación de los tipos compositivos, donde raramente hay coincidencias y cada autor proyecta su propio modelo de clasificación. Características comunes a todos ellos son tanto la dispersión teórica según la tendencia lingüística e incluso, a veces, según los autores, como la mezcla de consideraciones sincrónicas y diacrónicas, sin que muchas veces queden delimitadas claramente, aunque las últimas parecen necesarias para entender el análisis sincrónico de determinados compuestos ${ }^{8}$. Es decir, estos estudios muestran que la diacronía de la formación de compuestos incide de modos diversos en el análisis sincrónico, y se puede convertir en herramienta de la descripción linguiística de determinados compuestos. Son, asimismo, escasos los trabajos parciales sobre determinados aspectos o tipos compositivos. Tan sólo se han estudiado los más productivos, como las formaciones de verbo + complemento $^{9} \mathrm{o}$ de sustantivo $+i+$ adjetivo $^{10}$ y han sido poco trabajados otros.

Quizás se deba al hecho de que la composición no presente en romance el grado de desarrollo que manifiesta en otras lenguas, como las germánicas, el que casi siempre haya constituido tradicionalmente un campo periférico de la investigación linguiística en favor de otros aspectos de más envergadura como la derivación. Esto también explicaría la ausencia de un modelo teórico de análisis diacrónico. En general, podemos decir que, salvo en los pocos casos en que hay herencia directa del latín, los romances hubieron de reestructurar e innovar los esquemas de composición de palabras, desarrollando muchos patrones que eran incipientes en el latín vulgar, hasta superar en riqueza y variedad al propio latín. Luego, sobre esa base común ya romance, cada lengua fue evolucionando un esquema más que otro, según las necesidades expresivas y la adaptación a los respectivos sistemas lingüísticos. Por eso vamos a encontrar en la actualidad distintas soluciones en unos romances que son desconocidas en otros. Valdría la pena estudiar detenidamente este proceso ya no sólo en cada romance, sino también desde una óptica amplia panrománica, lo que no se ha hecho hasta el momento.

Madrid, Arco/Libros 1995, y Soledad Varela, Morfología léxica: la formación de palabras, Madrid, Gredos, 2005.

8 Véase Fernando González Ollé y Manuel Casado Velarde (1992), «Formación de palabras», Lexikon der Romanistischen Linguistik (LRL), Günther Holtus, Michael Metzeltin, y Christian Schmitt, eds., volumen VI, 1, Max Niemeyer Verlag, Tübingen, 1992, págs. 91-109: «La FP constituye uno de los ámbitos lingüísticos donde con mayor frecuencia y dificultad de interpretación se entre cruzan sincronía y diacronía en las operaciones de análisis etimológico y de análisis funcional para delimitar los constituyentes de una palabra» (pág. 94).

9 Por ejemplo el trabajo de Paul M. Lloyd, Verb-complement compounds in Spanish, Tübingen, Niemeyer, 1968 o el de Eugenio Coseriu, «La formación de palabras desde el punto de vista del contenido (a propósito de 'coupe-papier')», Gramática, semántica, universales, Madrid, Gredos, 1978, págs. 239-264.

10 Francisco García Lozano, «Los compuestos de sustantivo + adjetivo del tipo pelirrojo», Iberorromania, 7, 1978, págs. 82-89. 
Pensamos que un planteamiento estrictamente diacrónico del problema que nos afecta debe tener en cuenta los siguientes parámetros básicos u objetivos:

\section{CARACTERÍSTICAS DEL COMPUESTO}

La composición muestra límites incómodamente borrosos, que apenas se delimitan entre la morfología (por cuanto se podría considerar - y se considera tradicionalmente - un proceso morfológico de creación de nuevas palabras), la sintaxis (ya que son la manera en que determinados sintagmas se lexicalizan o el aprovechamiento de determinadas estructuras sintácticas para la creación léxica) y la lexicología (pues el resultado es siempre una nueva palabra, con determinadas características similares al conjunto de lexías de la lengua, aunque a diferencia de éstas, como veremos pronto, interviene en mayor grado la motivación en la creación del nuevo compuesto). Estos límites tienden a desdibujarse y entremezclarse confusamente mucho más desde un punto de vista de evolución histórica, lo que hace más complicada su formulación teórica.

Se ha de partir, en primer lugar, de una definición de la composición. La definición de compuesto tiene los mismos problemas que la definición de palabra ${ }^{11}$. Como se ha observado, la palabra compuesta es una realidad intermedia entre la lexía simple y el sintagma, de ahí que sean diferentes las direcciones propuestas en su estudio ${ }^{12}$. Sintácticamente, el compuesto se define como una unidad de funcionamiento dentro del enunciado, de tal manera que sólo puede ser sustituido globalmente por otras palabras o por perífrasis oracionales y la determinación que puede recibir afecta al compuesto entero, no a una de sus partes. Morfológicamente también hay compuestos que se aproximan a los grupos sintácticos más que otros; todo depende de su grado de aglutinación a lo largo de la historia. Más próximo al sintagma estarían las expresiones sintagmáticas del tipo lengua de gato, que en general no suelen sobrevivir como tales, pues la evolución histórica tiende a aglutinar los dos elementos (hoja de lata $>$ hojalata). En segundo lugar, se situarían los compuestos (o aposiciones) formados por dos sustantivos asindéticamente combinados que crean su plural sobre el primer elemento o núcleo: hombre(s) rana, buque(s) escuela. El tercer grupo estaría formado por aquellos compuestos en los que existe pluralidad de

${ }^{11}$ Véanse José Alberto Miranda, op. cit., págs. 7 y sigs., Eugenio de Bustos Tovar, «Algunas observaciones sobre la palabra compuesta como signo lingüístico», Revista de Filología Española, XLIX, 1966, págs. 255-274, y Fernando González Ollé y Manuel Casado Velarde, art. cit. págs. 103-104.

${ }^{12}$ Es imprescindible, en este sentido, el extenso trabajo de Eugenio de Bustos Gisbert, op. cit., quien a lo largo del libro ofrece una caracterización completa tanto de la composición como proceso de formación de palabras como de los distintos patrones o modelos de composición. 
soluciones, lo que evidencia su proceso de aglutinación: guardiaciviles - guardias civiles, casatiendas, casas tiendas, casastienda. Por último, estarían los compuestos en los que hay unión indiscutible de forma que el plural sólo afecta al conjunto y no hay problemas en considerarlos palabras y no sintagmas: carilargo, pelirrojo, gentilhombre, etc.

Desde un criterio semántico, aparece también una gradación que va desde la unidad referencial absoluta a la posibilidad de considerar al compuesto como un cuasi-sintagma en lo que a su contenido se refiere, con los consiguientes grados intermedios. El compuesto es una unidad significativa, que se caracteriza frente al grupo sintáctico, porque la información que nos proporciona es diferente de la deducible de la simple articulación de sus componentes en un grupo sintáctico correspondiente. Por ejemplo, puede ocurrir que la relación entre significado y referente se base bien en una comparación entre alguna cualidad del referente y alguno de los semas de los elementos del compuesto (patas de gallo), o bien en un proceso metonímico (abrecartas), o es necesaria una interpretación figurada, restrictiva o metafórica del modificador para comprender su contenido frente al grupo sintáctico correspondiente: mercado negro no hace referencia al color, sino que metafóricamente negro significa 'ilícito'. En estos casos, la motivación de la palabra compuesta resulta de la suma de la motivación morfológica, que como tales compuestos tienen, y de la motivación semántica que se desprende del uso figurado o metafórico de uno de sus elementos ${ }^{13}$.

La relación entre los constituyentes en el plano semántico es doble. Por un lado, se coincide en señalar relaciones sintáctico-semánticas entre los constituyentes del compuesto. Es fácil percibir que se establecen relaciones gramaticales (coordinación, subordinación y aposición), que son de naturaleza mucho más abstracta que las de la sintaxis; esto es, no dicen nada de la modalidad específica de esa relación ${ }^{14}$ que conviene tener en cuenta en su estudio. Por ejemplo, girasol = 'algo que gira y tiene relación con el sol', pero no se específica cual es esta relación ('girar alrededor, hacia, con el sol'). De ahí la concepción de la composición como «microtaxis» ${ }^{15}$ o como «gramática del léxico» ${ }^{16}$. Por otro lado, existen también relaciones semántico-referenciales del compuesto en conjunto en las que se basa la motivación. Estas relaciones tienen una importancia decisiva para comprender el compuesto, pues no basta la simple determinación de las relaciones sintáctico-semánticas, hay que buscar la motivación referencial en cada subsistema estudiado, o la consideración del referente de la designación.

\footnotetext{
13 Véase Bustos Tovar, art. cit., pág. 263.

14 Véase Eugenio Coseriu, art cit. y Fernando González Ollé y Manuel Casado Velarde, art. cit., pág. 104.

${ }^{15}$ En la concepción de Emile Benveniste, «Fundamentos sintácticos de la composición nominal», Problemas de lingüística general, II, Méjico, Siglo XXI, 1977, págs. 147-163.

${ }^{16}$ Así la considera Eugenio Coseriu, art. cit.
} 


\section{LA COMPOSICIÓN COMO PROCESO A LO LARGO DE LA HISTORIA Y SU RELA- CIÓN CON LA DERIVACIÓN}

El castellano tradicionalmente ha recurrido a los dos mecanismos conocidos para la formación de palabras mediante procedimientos morfológicos: la derivación y la composición. La parasíntesis no es considerada en sí misma un mecanismo específico de formación de palabras, sino la combinación de los diversos procedimientos de derivación o de derivación más composición. Otros añaden a esta lista los procedimientos de acortamiento y abreviación ${ }^{17}$, caracterizados por su corta y moderna existencia (cine, bus, Talgo) a diferencia de la derivación y la composición, que serían los procedimientos tradicionales desde los orígenes de la lengua.

Un estudio que aborde diacrónicamente la composición ha de intentar ofrecer una visión histórica de conjunto de la composición como proceso desde el latín hasta la actualidad. En primer lugar, en tanto que mecanismo, habría que distinguir la composición del mecanismo de la derivación (en lo que, por cierto, no hay unanimidad), con el que históricamente puede interferir, y ver de qué manera se han producido cambios en este sentido a lo largo de la historia. Esto es, podemos encontrarnos casos en los que un análisis sincrónico nos indica que se trata de un derivado que, diacrónicamente, tiene su origen en un compuesto. Lo mismo ocurre, desde el lado contrario, con lo que se denomina «derivación regresiva», cuando un compuesto puede surgir como producto de una regresión a partir de otro, como fotograbar, derivación surgida de fotograbado $^{18}$. Asimismo, habría que prestar atención a los casos fronterizos, por ejemplo, el de los compuestos con temas grecolatinos o con preposiciones. Así, determinadas palabras que en una época podrían ser compuestas pasan a ser consideradas derivadas en virtud de que uno de los componentes ha perdido su carácter independiente o no ligado, se ha lexicalizado, y pasa a ser un prefijo o un sufijo.

No entraremos aquí en el complejo problema de delimitación entre composición y derivación. Sin embargo, no podemos dejar de señalar las relaciones históricas entre ambos procedimientos. Remitimos a las consideraciones que a este respecto han hecho Wartburg ${ }^{19}$ Bustos Tovar $^{20}$ y Alvar y Pottier ${ }^{21}$. En

${ }^{17}$ Por ejemplo, Hernán Urrutia, op. cit. también Hernán Urrutia, y Manuela Álvarez, Esquema de morfosintaxis histórica del español, $3^{\mathrm{a}}$ ed., Bilbao, Universidad de Deusto, 2001, Fernando González Ollé y Manuel Casado Velarde, art. cit. y Alvar Ezquerra, op. cit.

${ }^{18}$ Véase J. F. Val, art. cit. pág. 4.760.

${ }^{19}$ Walther Wartburg, Problemas y métodos de la lingüística, Madrid, CSIC, 1951.

${ }^{20}$ Eugenio de Bustos Tovar, art. cit., págs. 255-274.

${ }^{21}$ Manuel Alvar y Bernard Pottier, Morfología histórica del español, Madrid, Gredos, 1983, págs. 361 y sigs. 
cuanto a su diferenciación respecto de la derivación mediante sufijos, Alvar y Pottier $^{22}$ consideran que la sufijación y la composición representan dos soluciones distintas a un mismo problema: el de la integración en el plano de la palabra de dos elementos de una construcción analítica. Se puede observar la afinidad existente entre, por ejemplo, cabrero y guardacabras. En uno y otro caso, los elementos que intervienen se pueden combinar de diversas maneras, según sean las respuestas que se deban dar a las cuestiones suscitadas. Podemos sobrentender el verbo cuidar, guardar, etc. y expresar el agente y el objeto, como ocurre en cabrero, o expresar el verbo y el objeto sin hacer mención del agente, como ocurre en guardacabras. La formación de palabras por composición es, además, más flexible que por derivación, pues se basa en los tipos habituales de estructuras sintagmáticas (sustantivo, adjetivo, verbo, complemento, etc.).

Para Wartburg ${ }^{23}$ no existe desde un punto de vista histórico diferencia entre composición y derivación. Un sustantivo puede desgastarse poco a poco semánticamente y degradarse hasta convertirse en sufijo. Por ejemplo, un caso límite entre compuesto y derivado son los adverbios españoles en -mente: mediante un desgaste semántico de mente (sustantivo) a -mente (componente de adverbio de modo). Wartburg señala que entre composición y derivación se establece una relación de continuidad histórica, de tal forma que se puede entender la derivación como una composición desgastada y extendida por analogía. Bustos Tovar $^{24}$ matiza esta afirmación de Wartburg, ya que no observa una secuencia necesaria que lleva de la composición a la derivación. Puede ocurrir, efectivamente, que la composición acabe siendo derivación, por ejemplo, la constante frecuencia de préstamos griegos y latinos tiene como consecuencia que lleguen a considerarse estos préstamos como semiprefijos o sufijos derivativos, ya que no existen como palabras independientes en la lengua. También parecen derivados los vocablos que encierran una palabra diacrítica, ya sea un préstamo, ya sea un arcaísmo, que existe sólo en el compuesto, como aguarrás, aguaducho (con el matiz despectivo de otros derivados en $-u c h{ }^{25}$ ) o susodicho.

Sin embargo, también hay que tener en cuenta que la evolución fonética deshace la individualidad fónica de los constituyentes hasta arruinar su motivación y dar lugar a una lexía simple, no necesariamente un sufijo (por ejemplo, BENE DICTO > bendito), o incluso al contrario, en virtud de un proceso más complejo (conocido como etimología popular), por afán de expresividad se puede intentar recobrar la pérdida de la motivación mediante la tendencia a analizar formas léxicas que podría llevar de la derivación a la composición (por ejemplo, es lo que ocurre en el paso de vagabundo > vagamundo). El compues-

\footnotetext{
22 Alvar y Pottier, op. cit., pág. 361.

${ }^{23}$ Op. cit., pág. 138.

24 Art. cit., págs. 269-270.

${ }^{25}$ Bustos Tovar, art. cit., pág. 265 y n. 6.
} 
to pasa a formar parte del léxico de la lengua como una unidad más, por lo que se va a ver sometido a los mismos procesos de desgaste fónico y semántico que cualquier otra palabra, de manera que se puede dar un proceso de desmotivación que culmina, en algunos casos, en la pérdida de conciencia del hablante del carácter del compuesto, su total lexicalización. Un caso ejemplar lo constituye hidalgo: fijo/a/s de algo $>$ fijo/a/s dalgo $>$ hijodalgo $>$ hidalgo $>$ hidalgo/a/s. La pérdida de motivación a lo largo de la historia puede explicar en muchos casos el paso de compuesto a derivado o a palabra simple en la que no se percibe la composición, y el afán de expresividad o motivación justifican la sustitución de un derivado por un compuesto. El contenido semántico del compuesto apunta hacia una única entidad referente, que explica por sí sola los procesos históricos de desmotivación o, al contrario, la reinterpretación como compuestos de unidades que no lo son en origen.

Por otro lado, sería interesante establecer las características fonológicas, morfosintácticas y semánticas que presentan los compuestos en español y ponerlas en relación con los cambios que se han producido en este sentido a lo largo de la historia tanto en el propio sistema como en otros romances. Como hemos visto antes, el concepto de composición implica un estadio intermedio entre palabra y frase. No en vano, podríamos describir la composición como una microsintaxis o la manera en la que la sintaxis se manifiesta en el componente léxico. Esto puede tener aspectos históricos muy interesantes que a nuestro juicio apenas se han destacado debidamente. De hecho, por ejemplo, hay palabras que tienen su origen en un compuesto antiguo donde han quedado fijado un modelo sintáctico hoy inexistente en romance, como por ejemplo, condestable (< COMITE STABULI), en que ha sobrevivido incluso la marca o forma del antiguo genitivo. Puesto que, como estamos viendo, los compuestos participan también de la sintaxis de un estado de lengua determinado en una época determinada, se puede esperar que si ésta cambia, incida a su vez en cambios en los patrones o modelos compositivos. Así, como vimos anteriormente, frente al latín, en el que el orden de los elementos presentaba normalmente la estructura determinante + determinado o objeto + verbo (RIVIANGULU $>$ riaño o MAGNU ANIMU > magnánimo), el romance tiende al orden inverso (hierbabuena, sacacorchos).

\section{MotiVACIÓN Y LEXICALIZACIÓN}

En su artículo sobre el compuesto considerado desde su motivación, Bustos Tovar $^{26}$ ofrece aspectos interesantes de los compuestos que se pueden poner en

${ }^{26}$ Art. cit. págs. 259 y sigs. 
relación con la diacronía de su proceso de formación. Precisamente su carácter de elemento motivado es el que nos va a permitir otra aproximación histórica a su estudio. Como ha estudiado Bustos Gisbert ${ }^{27}$, existe una gradación que va desde los compuestos muy cercanos al sintagma hasta las palabras que han perdido este carácter por su total lexicalización. Esta gradación se corresponde con el grado de motivación del compuesto. Cuanto mayor sea la motivación, más cerca nos encontramos del sintagma nominal de donde muchas veces procede (su valor expresivo está íntimamente ligado a la condición de analizable por parte del hablante). Cuanto mayor sea su lexicalización, más cerca estaremos de la palabra. La motivación alude, pues, a la relación de necesidad que el hablante establece entre una palabra y su significado, o entre una palabra y otro signo. Todo léxico cuyas voces normales son analizables formal o semánticamente por el hablante es motivado, lo que se relaciona estrechamente con la expresividad. De este modo, sería motivado un compuesto como capisayo en el que es posible distinguir la motivación morfológica que tiene en cuanto compuesto y la motivación semántica que nace de la suma de los significados de los dos términos en una única representación que afecta al conjunto no a los elementos que lo forman ${ }^{28}$. Son inmotivados, en cambio, los vocablos no analizables por el hablante (capa, sayo), que deben su significado a la tradición sólo. Por otro lado, consideramos con L. Ruiz la lexicalización en un sentido restrictivo, como «la pérdida de propiedades fundamentalmente gramaticales en beneficio de la fusión de varios elementos en una sola palabra, en una sola unidad simple tanto léxica, como gráfica» ${ }^{29}$, como se puede observar en el paso de hoja de lata > hojalata. Por tanto, motivación y lexicalización están relacionados, pero en sentido inverso: cuanto mayor sea la motivación, menor será su lexicalización, y viceversa.

\section{PREFERENCIAS Y EXPRESIVIDAD}

Bustos Gisbert ${ }^{30}$ ha puesto de manifiesto la importancia de las consideraciones del hablante, sus prejuicios o su deseo de expresividad, ya no sólo para explicar la creación de un determinado compuesto, sino también para poder analizar las relaciones semántico-referenciales que ayudan a entender su significado.

Parece haber también una mayor carga expresiva en varios de los esquemas de composición frente a la derivación. La expresividad, por su parte, que está

\footnotetext{
27 Op. cit. págs. 19 y sigs.

28 Bustos Gisbert, op. cit., pág. 263.

${ }^{29}$ Leonor Ruiz, Aspectos de fraseología teórica española, Anejo XXIV de Cuadernos de Filología, Valencia, Universitat de València, 1997, pág. 97.

${ }^{30}$ Op. cit.
} 
en el origen de muchos compuestos, se vincula también muy estrechamente con la motivación, de tal manera que una mayor lexicalización supone también una pérdida de expresividad. Mediante la expresividad del compuesto, se podría explicar, por ejemplo, la aparición de determinados tipos compositivos en unas épocas literarias, frente a su escasez en otras. Esto supondría entender también por qué determinados esquemas compositivos son más propios de la oralidad mientras que otros esquemas y buena parte de los derivados lo son de la escritura, sin que queden delimitados claramente algunas veces o se produzcan trasvases o influencias entre una y otra según las épocas.

Esto lleva a otra consideración de diferenciación expresiva entre composición y derivación. Es significativo, en este sentido, que la composición, por su misma fuerza expresiva, se vincula y se adapta mejor al discurso oral que al escrito, que prefiere la derivación. En la composición hay mucha mayor libertad para el hablante y son más frecuentes las creaciones expresivas y ocasionales. Como ha señalado Bustos Tovar ${ }^{31}$, en español la motivación es mucho mayor en la composición que en la derivación. La motivación relativa del compuesto español es más intensa y eficaz que la propia de la derivación. Este carácter de palabras motivadas en su significado explica su particular frecuencia en el lenguaje afectivo en las diversas épocas del idioma, con valores muchas veces despectivos, humorísticos o irónicos ${ }^{32}$. Este valor expresivo de muchos compuestos nace del hecho mismo de la composición: la suma de significados de los dos términos crea una representación sensorial en la que se basa la motivación semántica que afecta a la unidad léxica, no a los elementos que la forman independientemente. Dentro del plano de la motivación expresiva hay una gradación que va desde palabras motivadas fónicamente (como las onomatopeyas) hasta la mínima motivación morfológica de los derivados, lo que ha sido confirmado repetidamente por la lingüística histórica.

También debemos tener en cuenta que ha habido épocas de la lengua en las que se ha producido mayor entrada de compuestos que en otras. Por ejemplo, recordemos lo que supuso la incorporación de las tierras americanas con un Nuevo Mundo lleno de objetos, animales y flora que hasta entonces no tenían nombre en castellano. Se debía dar nombre a lo que, por desconocido, no se poseía en la cultura europea. Para ello, junto con el préstamo de las lenguas indígenas, se echó mano de la tradición y de los propios significantes de la lengua para designar nuevos referentes. Un recurso abundante fue la formación de palabras mediante derivación sufijal, pero no escapó a las necesidades expresivas la fuerza de muchos compuestos que se fueron incorporando a lengua en

\footnotetext{
31 Art. cit., pág. 262.

32 Véase Francisco Ynduráin, «Sobre un tipo de composición nominal verbo + nombre», Presente y Futuro de la Lengua Española, II, Madrid, 1964, págs. 297-302.
} 
gran número. Mediante el compuesto se aseguraba la referencia precisa de lo designado, sobre todo ante aquellos que nunca habían visto previamente el referente $^{33}$.

\section{EL COMPUESTO COMO PALABRA}

Se han de especificar los procesos generales históricos que afectan al compuesto, en tanto que signo lingüístico que sufre los mismos avatares que cualquier elemento léxico, de manera que puede ser objeto de estudio de la lexicología. Como acabamos de ver, el compuesto es un signo motivado surgido de determinadas necesidades expresivas y de denominación que, una vez se inserta en el léxico, sigue los mismos procesos de desgaste que producen su total lexicalización. También puede darse la presencia de palabras diacríticas entre los elementos integrantes del compuesto, lo que incide también de manera directa en la pérdida de la motivación y de la expresividad que pudo haber sido causa de su origen. Por ejemplo, la voz murciélago (< MUR CAECUS), desde un punto de vista actual de análisis sincrónico, o desde la perspectiva del hablante, no sería un compuesto. Históricamente lo es, lo que ocurre es que el primer elemento, mur 'ratón' en latín, ha desaparecido en castellano. La conversión en diacrítica de una voz esta relacionada con multitud de factores que interesan a la lexicología.

A esto se añade que no se puede deslindar sin más el compuesto, en tanto que palabra o lexía nueva, de la historia del léxico de una lengua. En este sentido, en la palabra compuesta también se pueden operar cambios semánticos, como el que llevó a aguaducho (< AQUAEDUCTO) a su sentido de 'puesto de venta de agua' ${ }^{34}$, quizás por su completa lexicalización. Es más, como pieza léxica se inserta en el nivel del sistema más expuesto a los avatares externos, como las modas, las preferencias de los hablantes, la aparición de determinadas circunstancias, etc. Es decir, es necesaria la consideración del compuesto también desde la perspectiva de la historia externa de la lengua a través de la lexicología. Los mismos hablantes, por razones diversas dada la expresividad de muchos compuestos, no sólo pueden intervenir en la preferencia por un determinado modelo compositivo sobre otro, sino que pueden alterar también la morfología o el patrón compositivo. Recordemos, por ejemplo, como la aparición sistemática de - $i$ - en determinados compuestos como pelirrojo a partir del siglo XV se ha explicado mediante una tendencia cultista del humanismo. En la

${ }^{33}$ Véanse Tomás Buesa y José María Enguita, Léxico del español de América. Su elemento patrimonial e indígena, Madrid, Mapfre, 1992, pág. 172.

${ }^{34}$ Véase Joan Corominas y José Antonio Pascual, Diccionario Crítico Etimológico Castellano e Hispánico, Madrid, Gredos, 6 vols. 1980-1991, s. v. agua. 
misma perspectiva hay que situar el origen de tiquismiquis (a partir de latín macarrónico tichi michi, alteración vulgar de tibi, michi, a partir de mihi ${ }^{35}$.

\section{HistORIA DE LOS DISTINTOS PATRONES O MODELOS COMPOSITIVOS}

Gran parte del estudio diacrónico de la composición consiste en establecer previamente los distintos tipos compositivos (lo que es tema muy controvertido entre los autores) y sus características a lo largo de la historia de la lengua. Esto implica la adopción de un aparato teórico que permita establecer unos criterios coherentes de clasificación y análisis de los compuestos y supone también buscar modelos de evolución de estos tipos. Aquí deberían establecerse cuáles han sido los más y los menos productivos según épocas o etapas históricas, buscando las causas internas y externas que los expliquen. También habría que dar cuenta de los modelos de composición que en una etapa son escasos y en otra etapa son abundantes.

La formación de palabras por composición ha estado presente en todas las épocas de la historia de la lengua desde los orígenes, si bien en castellano la predominante ha sido la composición nominal, dada la escasa rentabilidad y productividad que muestra la verbal. Algunos de los patrones o modelos de composición los heredará directamente del latín (como MANU-MITTERE 'dar libertad al esclavo', CAPITI-LAVIUM 'loción de la cabeza del recién nacido', RUINA MONTIUM, 'procedimiento minero para la extracción del oro mediante el derrumbe de una montaña'), otros serán un desarrollo románico posterior, que el castellano comparte con el resto de romances en mayor o menor grado. A esto se añade que hay esquemas compositivos que fueron preferidos en todas las épocas sobre otros, que sólo destacaron en algún momento o siempre fueron minoritarios; asimismo, algunos han sido más comunes en unos ámbitos, como el literario, y otros propios de la lengua hablada de todos los tiempos. Muchos de ellos muestran una historia particular respecto de los otros en la que intervienen factores de todo tipo. En general, los patrones compositivos del español se corresponden con los de los demás romances, si bien presenta algunas características propias desconocidas en otros romances (pelirrojo, sombripuesto).

\subsection{La composición nominal}

Dentro de este tipo, para su estudio y clasificación, y siguiendo a Cano Aguilar ${ }^{36}$, podemos distinguir los casos en los que la estructura interna del

\footnotetext{
${ }^{35}$ Diccionario de la Real Academia Española, Madrid, Espasa Calpe, 1992, s. v. tiquismiquis.

${ }^{36}$ Rafael Cano Aguilar, El español a través de los tiempos, Madrid, Arco/Libros, 1988, págs. 189-191.
} 
compuesto no refleja ningún tipo sintáctico propio de la lengua (aunque pueda conservar, como veremos, algún tipo ya desaparecido), y aquellos otros en que la composición no hace sino convertir en unidad léxica lo que antes era una secuencia sintáctica.

6.1.1. Compuestos nominales que no reflejan ningún tipo sintáctico propio de la lengua

En estos compuestos es donde con mayor facilidad puede reconocerse la composición, de ahí que también se la denomine «composición propia» ${ }^{37}$. Sin embargo, como veremos, desde el punto de vista histórico, no se puede establecer una separación tajante entre los compuestos sintagmáticos y determinados tipos de composición «propia», por cuanto ciertos casos pueden ser el resultado de un proceso de aglutinación, como por ejemplo, agua de sal > aguasal.

Podemos distinguir dos patrones de composición presentes en el castellano de todas las épocas: a) los compuestos sustantivos formado por sustantivo + sustantivo (tipo zarzamora) y los compuestos adjetivos formados por sustantivo $+\mathrm{i}+$ adjetivo (tipo boquirroto). Pasemos a los primeros.

\subsubsection{Compuestos de sustantivo + sustantivo}

Este tipo compositivo ya estaba presente en latín vulgar, si bien era raro (por ejemplo, ARCUBALLISTA 'ballesta combinada con un arco'). Su desarrollo se alcanzó ya en época romance. Hay ejemplos antiguos en castellano, como malvavisco (< MALVA HIBISCU), puercoespín, picobarreno o varapalo. Es evidente que la tendencia integradora es más acusada en los compuestos más antiguos y de uso más habitual.

Son poco frecuentes en español y, en general, en todas las lenguas románicas. Presentan un mayor grado de integración entre los constituyentes que los sintagmáticos, en el que el compuesto puede mostrar un proceso de aglutinación o cruce de sus unidades léxicas, con pérdida de sustancia fónica, normalmente del primer elemento (coyotomate). Sin embargo, para algunos estudiosos secuencias del tipo buque escuela, pájaro mosca, empresa piloto, etc., serían aposiciones o estructuras lexicalizadas en las que el primer elemento se ve variado por el segundo. Este segundo elemento supone una indicación especial

${ }^{37}$ Es la denominación tradicional que aparece en el apartado dedicado a la composición de las Gramáticas de la RAE, Real Academia Española, Gramática de la lengua española, Madrid, Espasa-Calpe, 1931 y Real Academia Española, Esbozo de una gramática de la lengua española, Madrid, Espasa-Calpe, 1973. 
respecto al primero. Es una modificación del concepto, pero es también una calificación, ya que se están convirtiendo en adjetivos en virtud de la habilitación o translación. En todo caso, se reconoce el hecho de que se trata de tipos híbridos en los que aún no se ha dado la lexicalización completa (por ejemplo, en la marca de número, que sólo aparece en el primer término: peces espada).

Vemos que el segundo elemento es una aposición con valor adjetivo: el primer elemento suministra la denominación y el segundo especifica al primero, adhiriéndole el nombre de otra clase, lo que ha sido puerta de entrada a creaciones humorísticas. El objeto designado de esta forma no participa idénticamente de las dos clases semánticas que se le atribuyen. Como explica Benveniste $^{38}$, a una pertenece por naturaleza, a otra es atribuido figuradamente, por ejemplo, un pájaro mosca es un 'pájaro' con alguna semejanza con una 'mosca'. Esta relación puede hacer más abstracta y llegar incluso, en su motivación variada, a usos metafóricos del elemento determinante, como ocurre en hombre rana (quizás por las aletas de los buceadores que recuerdan a las de la rana).

Podemos distinguir distintos grupos de estas formaciones desde un punto de vista diacrónico:

a) Hay un grupo de palabras en las que se puede rastrear su origen en compuestos donde el primer constituyente actúa como elemento complementario; mantienen, así, la estructura y el orden latinos en que el nombre en genitivo precede al núcleo. A ellos responden formas como aguaducho $(<\mathrm{AQUAE}-$ DUCTO), pezuña (< EDIS UNGULA) o los más tardíos casapuerta, zarzamora, ferrocarril. En todo caso, estos ejemplos en que el elemento complementario precede al núcleo han sido en la historia tan raros como escasos. Esto puede en parte explicar por qué en la mayoría de estas formas, se ha producido ya su total lexicalización. Esta pérdida de motivación puede llevar incluso al desuso de la palabra o a su simple sustitución por un préstamo. Es lo que ha sucedió, por ejemplo, con la anterior voz, ya simple y no compuesta, aguaducho (< AQUADUCTU). Hoy ha quedado anticuada y sólo sobrevive en Andalucía con la acepción de 'puesto para vender agua', acepción muy separada de su primer sentido, lo que demuestra la pérdida de motivación semántica que debió sufrir ya en época temprana. En todo caso, desde el siglo XVII fue sustituida por el cultismo acueducto.

b) También puede ocurrir que el compuesto de sustantivo corresponda a una construcción preposicional no manifiesta en él. Se sobrentiende una preposición que indicaba la subordinación. Presentan una construcción sin correlato en el campo de la sintaxis, o una trasgresión de las normas sintácticas, al suponer la elisión de una preposición como fase previa a la formación del compuesto. Son los casos de aguamanos ('agua de manos'), aguamanil, salpresa

\footnotetext{
${ }^{38}$ Benveniste, art. cit., pág. 151.
} 
(< SALPRESU), etc. o los pocos ejemplos en que aparece una $-i$ - como marca de composición, producto de la aglutinación de los dos elementos, como cornicabra, cachicuerno, etc. Alvar Ezquerra ${ }^{39}$ considera estos tipos como restos del caso oblicuo latino. En este grupo pueden incluirse también aquellos casos en que podría haberse perdido una antigua preposición de intercalada. Es decir, que tienen su origen en sintagmas preposicionales (frecuentes en todos los tiempos del idioma) que han pasado a funcionar como una unidad, lo que ha facilitado su integración fonética, que hace ocultar la construcción gramatical originaria (lexicalización), por ejemplo maestrescuela y luego maestresala, tela de araña > telaraña. En los topónimos es especialmente abundante, como los citados por Menéndez Pidal ${ }^{40}$ Adealpozo, Majaelrayo, Puente la Reina, etc.

c) En otros ejemplos es todo el compuesto originario el que parece implicar una relación sintáctica con elemento no explícito, como ocurre en cabelprieto ('de cabello prieto') y en todos los topónimos que heredan un genitivo dependiente de un nombre genérico (así, (ECCLESIA) SANCTI FACUNDI > $S a(n)$ $(f) \operatorname{agun}(d))$.

Dadas estas características, su incidencia y su valor a lo largo de la historia de la lengua han sido desiguales. En general, su productividad ha sido mayor en algunos ámbitos como el literario que en otros, como el propio del coloquio. Ha sido un tipo de patrón de construcciones apositivas equivalentes a símiles o metáforas muy del gusto de la literatura barroca como podemos ver en los ejemplos que nos proporciona Lapesa ${ }^{41}$ : «Truxeron toros leones (fieros) para hércules (fuertes) cavalleros» (Lope); «conocimientos linces» (Tirso), «voces sirenas» (Tirso). Dama duende, nariz sayón, clérigo cerbatana. También se han dado, aunque menos, en la literatura de otras épocas: el hombre gas y el hombre globo de Larra, o, en nuestro siglo, menudean en la prosa de Ortega y Gasset (ideas-creencias, hombre-masa). En el siglo XX alcanzan mayor productividad, bien por la gran difusión de expresiones de uso internacional (hora punta, ciudad jardín, coche cama); bien por la frecuente aplicación de un mismo segundo elemento (empresa piloto, piso piloto, prueba piloto, etc.). El lenguaje publicitario ha incrementado considerablemente este recurso (precio ocasión, rebaja aniversario), junto con la lengua oral con fines humorísticos (hombre ladrillo).

\footnotetext{
39 Op. cit., pág. 31.

40 Op. cit., pág. 239.

${ }^{41}$ Rafael Lapesa, Historia de la lengua española, 9ª ed., Madrid, Gredos, 1981, págs. 355 y sigs.
} 


\subsubsection{Compuestos de sustantivo $+i+$ adjetivo}

Constituyen un tipo de composición muy característico en español. Este es el sistema compositivo adjetivo más importante: cuantitativamente ha sido muy productivo en español desde siempre; cualitativamente es el tipo de compuestos que más problemas suscita en cuanto a su interpretación semántica. También tiene un carácter típicamente hispánico, frente a las soluciones de otras lenguas románicas. Todo esto ha contribuido a que sea también un sistema que ha merecido estudios detallados, como el de García Lozano ${ }^{42}$, que señala restricciones interesantes en la forma y la relación sintáctica y semántica de estos compuestos.

Son compuestos muy raros en el período latino y bajolatino y parecen ser desarrollos romances posteriores. Una característica importante de estos compuestos es que es el sustantivo el que aparece subordinado al adjetivo, y no al revés, de donde se deriva también el sentido posesivo que se sobrentiende, como observa Menéndez Pidal ${ }^{43}$ en el caso de boquirrasgado, 'que tiene la boca rasgada'. En la interpretación sintáctico-semántica de estos compuestos, el adjetivo es el núcleo semántico de la expresión compleja, y el sustantivo lo determina, lo que se ha interpretado como un ejemplo de lo que se ha denominado «acusativo griego» o «acusativo de relación». Según Lapesa ${ }^{44}$, a quien seguimos en esta explicación, con este nombre se entienden dos tipos diferentes de construcciones:

1. En primer lugar el acusativo de relación o parte, normal en griego y adoptado por los poetas latinos desde época imperial (NUDA GENU). Se considera un artificio sabio y erudito que no tuvo en latín raigambre popular. Fue posteriormente imitado por la literatura culta del Renacimiento. En España hay un influjo clásico y petrarquista; entra en nuestras letras como complemento sin preposición con Garcilaso («los alemanes / el fiero cuello atados») y sigue apareciendo en el Barroco, como en Góngora: «desnuda el brazo, el pecho descubierta». Pero no dejó de ser nunca un uso artificial sin trascendencia.

2. Junto a este empleo erudito y rebuscado parece haber habido en latín una tendencia espontánea a la creación de un acusativo de relación o parte a través de construcciones de doble acusativo especialmente abundantes en el latín de los siglos VI al VIII. En esa tendencia parecen tener su origen este tipo de compuestos románicos cuyo primer elemento es un sustantivo que indica la parte del cuerpo o del vestido a que se refiere un participio o adjetivo: tiest he-

\footnotetext{
${ }^{42}$ Art. cit. pág. 83.

${ }^{43}$ Op. cit. pág. 240.

${ }^{44}$ Rafael Lapesa, «Los casos latinos: restos sintácticos y sustitutos», Estudios de morfosintaxis histórica del español, Madrid, Gredos, Vol I, 2000, págs. 73-122, pág. 103 y sigs.
} 
rido, cuello albo, barbapuniente, picoabierta, cabeztuerto, que era la forma habitual del acusativo de relación. A partir del siglo XV, como veremos, se generalizó la modificación del primer elemento con la inserción de la -i-.

Por lo tanto, semánticamente el segundo elemento del compuesto predica una cualidad del primero (cejijunto es 'junto de cejas') o el estado resultante de un proceso bien directamente (boquiabierto, 'asombrado'), o de manera figurada, como en alicaído o patidifuso. En todo caso, este tipo de formaciones está muy restringido respecto a su aplicabilidad, por cuanto se limitan al campo referido a personas y animales. Su función habitual es la de ser adjetivos, pero, en el caso de las designaciones de animales, es frecuente observar como se producen sustantivaciones cuando la característica descrita por el compuesto es interpretada como esencial por parte del hablante, como en rabipelado, cabecinegro, petirrojo, cariblanco, etc. Muchos de estos compuestos aparecen con profusión en los Cronistas de Indias cuando describen la fauna y flora de América. También encontramos lexicalizaciones en formaciones antiguas en que los sentidos originarios de los componentes ya no se perciben, o están muy lejanos: casquivano, peliagudo ${ }^{45}$.

Una cuestión que plantean estos compuestos en el que se combinan temas de carácter diacrónico, semántico, sintáctico y morfológico es el de la presencia del interfijo - $i-$, como marca más caracterizadora de su morfología. Desde el punto de vista diacrónico, ya en el siglo XIII se halla algún caso de la unión de ambos elementos por $-i$-: barbirrapado ${ }^{46}$, pero hasta el siglo XV era frecuente que estos compuestos se produjeran sin la intercalación de ningún elemento, y, así, los dos elementos solían mantener su integridad fónica: bocabierto, bocarrasgado, picoabierto ${ }^{47}$. Sin embargo, a partir del siglo XV se imponen la formas con -i-, como en patitieso, zanquituerto. Para la mayoría de los estudiosos el interfijo se toma por imitación del latín en construcciones excepcionalmente frecuentes del tipo ALTI TONANS, BARBIRASU, PLENILUNIU, en las que aparecía una - $i$ - de transición; esta - $i$ - podría ser un resto sintáctico o una reposición culta o semiculta. Se trataría de restos latinizantes en formaciones vulgares, inexistentes en latín, pero creadas en español por analogía con otras, ya que la documentación más antigua carecía de esa - $i$ - como hemos visto. Esta tendencia se puede encontrar en el deseo de los poetas cultos del siglo XV de dotar a la lengua de ornamento semejante al epíteto griego compuesto, que llevó a formaciones del tipo clarífico, belígero (Juan de Mena, Laberinto), bicípit, imbrífero (Juan de Castellanos), etc. Se trata de un tipo de composición con evidentes pretensiones de cultismo. El aspecto de latinismo lo daba la presencia de

\footnotetext{
45 Véase Alvar Ezquerra, op. cit., pág. 32.

46 Véase Cano Aguilar, op. cit. pág. 189.

${ }^{47}$ Menéndez Pidal, op. cit., pág. 240.
} 
esa $-i$-, que hacia pensar en algo como un genitivo latino ${ }^{48}$. En tal sentido se ha interpretado que en formaciones como aliabieto, barbiponiente o manilargo ha influido el latín humanístico.

Tanto la literatura como la lengua popular se han servido de este modelo para la creación de compuestos más o menos expresivos, muchos de carácter ocasional. Baste recordar, a modo de ejemplo, de las posibilidades de este tipo de composición puestas al servicio de la expresión en el Diálogo de vida beata (1563) de Juan de Lucena ${ }^{49}$, en el que siguiendo el gusto de su tiempo, creará compuestos de aire culto como ornígero, o auripenado; pero también recurrió a menudo a combinaciones léxicas, algunas con tradición desde Berceo y otras creadas ad hoc: como artifecho ('hecho con arte'), que opone a natural, o incluso utiliza como parte de un infinitivo compuesto una forma consolidada en la lengua como adjetivo: «Mi culpa, señor obispo, mi culpa es haverme boquirroto contra ti».

Por otro lado, se ha observado una tendencia a que el primer constituyente del compuesto sea bisílabo y terminado en vocal ${ }^{50}$. Sin embargo, Montes Giraldo $^{51}$ ofrece ejemplos colombianos que muestran el debilitamiento de esta tendencia a las dos sílabas.

6.1.2. Compuestos nominales que aprovechan las estructuras sintácticas de la lengua

Este tipo de compuestos surgen de la conversión en una unidad léxica de lo que previamente había sido un sintagma. Puede ocurrir, como observa Menéndez Pidal que «varias palabras unidas conforme a leyes sintácticas ordinarias, por usarse así unidas vinieron con el tiempo asoldarse, cambiando su imagen por otra simple y única» ${ }^{52}$. Pero su origen también puede ser el aprovechamiento de una determinada estructura sintáctica o enunciado para crear una nueva lexía compuesta. Las clasificaciones que se han hecho de esta clase de compuestos se han basado, en general, en el tipo de unidades funcionales que entran en la combinación, criterio que también seguiremos aquí.

48 Alvar y Pottier, op. cit., pág. 412.

49 Véase Javier Herrero Ruiz, «Formación de palabras en el Diálogo de vita beata de Juan de Lucena», Actas de III Congreso Internacional de Historia de la Lengua Española, Alegría Alonso González, coord., Madrid, Arco/Libros, I, 1996, págs. 349-355.

50 Val, op. cit., pág. 4817.

51 José J. Montes Giraldo, Compuestos nominales en español contemporáneo de Colombia, Bogotá, Instituto Caro y Cuervo, 1968, pág. 25.

${ }^{52}$ Op. cit., pág. 241. 


\subsubsection{Compuestos de sustantivo + adjetivo o adjetivo + sustantivo}

Este tipo de compuesto ha sido siempre uno de los más habituales en castellano. Constituyen un esquema de formación presente a lo largo de la historia de la lengua. Eran habituales en latín (AVETARDA > avutarda, RESPUBLICA, ROSMARINUS > romero, MAGNUS ANIMUS > magnánimo). Muestra, como en latín, la concordancia de ambos elementos. Algunos de estos compuestos son muy antiguos, tanto en la secuencia sustantivo + adjetivo: nochebuena, aguardiente; como adjetivo + sustantivo: buenaventura, malandança, mediodía, rico omne, etc. A este tipo podrían unirse los casos en que el elemento inicial es un determinante, como trébedes, o cien(to) pies. Ofrecen, por tanto, muestras de diversas etapas de la evolución linguística, en las que se puede observar la modificación o la pérdida de sustancia fónica (acompañada de progresiva lexicalización), por ejemplo, pimpollo (pino + pollo), vinagre (con apócope de -o en ambos casos), leo(n)pardo o pavipallo, que significa 'pollo joven', en el que la $-i$ - es difícil de explicar ${ }^{53}$.

Asimismo, hay palabras en las que casi se ha perdido completamente la motivación y ofrecen un alto grado de lexicalización, a pesar de que las unidades presentes sean parcial o totalmente reconocibles. En estos casos, la evolución fonética ha deshecho la individualidad fónica de uno (o de los dos) constituyentes del compuesto, con lo que se arruina su motivación. Un ejemplo con el que ilustran este proceso Alvar y Pottier ${ }^{54}$ es el de avutarda (< AV(E) TARDA) a través de abtarda y autarda: la - $u$ - se explica por epéntesis de /b/ antihiática, pues el influjo de ave hubiera configurado formas como avetarda (que siguen existiendo) en las que la etimología es transparente, mientras que en avu- seguiría alejada de la motivación ave. Su propio carácter compuesto puede intervenir en su fijación fónica a lo largo de la historia, por la pérdida de la conciencia por parte del hablante de su carácter compuesto, lo que es particularmente frecuente en innumerables topónimos, Castilblanco, Torquemada, etc.

Ahora bien, no necesariamente la pérdida de motivación de los compuestos más antiguos se debe sólo a la evolución fonética, en el mismo sentido puede operar la pérdida o desuso de uno de los términos, como vimos que ha ocurrido con murciélago. Su origen es el compuesto latino MUR CAECU ('ratón ciego') que dio en castellano murciego y su ampliación en murciégalo (que figura en Juan Manuel y hoy sigue vivo en algunas hablas). El primer compuesto mur convivió junto a ratón durante la Edad Media ${ }^{55}$. Todavía Valdés considera un

\footnotetext{
53 Ibíd.

54 Op. cit., págs. 415-416.

${ }^{55}$ Corominas y Pascual, op. cit., s. v. mur.
} 
asunto de comodidad estilística la elección entre ambos términos; sin embargo, en su época mur ya debía de estar en retroceso, pues en 1601 Francisco del Rosal reconocía que era voz anticuada. Con la pérdida de mur se arruinó para siempre la motivación semántica junto con la morfológica, atestiguada en la metátesis que concluirá en murciélago (documentada ya en 1251 en el Calila e Dinma). Ya no se percibe el antiguo sentido de «ratón ciego». La desaparición de mur ha oscurecido la motivación semántica y morfológica del compuesto.

Dentro de este tipo se han de incluir también las lexicalizaciones completas de determinados sintagmas o usos, como buenas noches, nombre con el que se conoce un determinado tipo de planta americana que se abre al anochecer. Otros compuestos de este tipo son más recientes y, por tanto, sus elementos no suelen presentarse soldados o presentar las dos posibilidades: campo santo / camposanto, guardia civil / guardiacivil, de ahí que también muestren ambas posibilidades en la marcación del número y el género: guardias civiles / guardiaciviles.

\subsubsection{Compuestos de sustantivo + sustantivo o adjetivo + adjetivo}

La unión de dos sustantivos o adjetivos puede remontar a una antigua aposición ${ }^{56}$, como ocurre en musaraña. Del latín MUS ARANEU o 'musgaño', así llamada por la creencia popular en el carácter venenoso de su mordedura, surge musaraño (conservada en portugués), que el castellano adaptará con la terminación araña. Otros casos son piedra sufre, cañaferla o cañaherla (< CANNA FERULA), salmuera (< SALE MURIA). En la combinación de adjetivos, además, la unión puede realizarse directamente, como en sordomudo, en el que la -o es invariable en el primer elemento (hispano-suizo/a).

Sin embargo, también es muy abundante el origen de estos compuestos en una coordinación, reflejada en la -i- intercalada entre los radicales, que muchos, consideran la conjunción coordinativa $y$, como se observa en compuestos antiguos y modernos del tipo capisayo, arquibanco, artimaña, carricoche, ajipuerro, agridulce, tontiloco, altibajo o pinto y parado (ya en el siglo XV). Se ha señalado que compuestos como agridulce o baciyelmo sirven para denotar realidades híbridas. Con sustantivos como capisayo, salpimienta se hace referencia a una realidad intermedia sin que podamos considerar a ninguno de los dos como núcleo. Con adjetivos como agridulce se expresa una cualidad también intermedia.

Los compuestos con dos adjetivos cuentan con amplísima productividad y muy poca o ninguna fijación léxica (lo que explicaría el hecho de que los dic-

${ }^{56}$ Véase Cano Aguilar, op. cit., pág. 189. 
cionarios recojan muy pocas unidades). Lo característico de este sistema compositivo es que la relación semántico-sintáctica existente es de carácter coordinativo, es decir no hay subordinación de uno de los elementos al otro, a excepción de algunos compuestos como verdinegro, donde hay subordinación pues su significado es 'verde oscuro'. Pueden caracterizarse por su antonimia (altibajo, claroscuro); designar color (rojinegro, blanquinegro), referir grupo cultural o nacional (anglosajón, latinoamericano), aludir a términos técnicos o científicos (lingüístico-cultural), o algunos ejemplos al margen de estos cuatro grupos como tontiloco, tontivano, etc. y poco más, pues este patrón se reduce a estos campos específicos.

\subsubsection{Compuestos de verbo + complemento, verbo + verbo y adverbio + verbo}

Era un subsistema muy raro en latín. Sólo en latín vulgar aparecía en algunos nombres propios. Se desarrollará con gran vitalidad en todos los romances y es uno de los sistemas compositivos más frecuentes en español de todos los tiempos. Lloyd ${ }^{57}$ muestra que se trata de un sistema de composición típicamente romance que se desarrolla a partir del siglo IX, con ejemplos aislados en los siglos IV y VIII. Desde entonces goza de gran productividad, lo que se podría explicar por la simplicidad y sistematicidad del propio sistema compositivo, a lo que se añade como señala la mayoría de los estudiosos la comodidad definitoria para el hablante de la semántica de estos compuestos. Se trata también del sistema más estudiado por los romanistas, sobre todo por su marcado paralelismo con el sintagma verbal oracional. Por eso es también donde más polémicas e interpretaciones se han producido a la hora de analizar su estructura y forma ${ }^{58}$.

Un primer problema que ha suscitado este tipo de compuestos ha sido el de la determinación de cuál es la forma presente en el constituyente verbal, para lo que no existe opinión unánime. Algunas interpretaciones lo consideran imperativo ${ }^{59}$,

57 Op. cit. págs. 13 y sigs.

${ }^{58}$ Para el español puede consultarse la historia y desarrollo de este modelo en Lloyd, op. cit., págs. 26 y sigs., quien clasifica los compuestos de este tipo a partir de las relaciones entre compuesto y designado, atendiendo a si designa a una persona, un animal, una cosa, una acción, etc.; pueden verse también Manuel Alvar Ezquerra, «De nuevo sobre los compuestos de verbo más sustantivo», II Simposio Internacional de la Lengua Española, Manuel Alvar Ezquerra, coord., Las Palmas, Cabildo Insular de Gran Canaria, 1984, págs. 83-98 y el artículo citado más atrás de Coseriu.

${ }^{59}$ Esta ha sido la opinión general de las primeras propuestas de romanistas (especialmente para el ámbito del francés, como Diez o Meyer-Lübke). Véase Lloyd, op. cit. págs. 3-10 para un resumen de las distintas posiciones al respecto. Una revisión más moderna se puede consultar en J. F. Val, art. cit., págs. 4.788 y sigs. 
otras como un presente de indicativo ${ }^{60}$ y para la mayoría es un mero tema verbal $^{61}$.

Parece que está clara la presencia del imperativo en compuestos como tentempié, vaivén, salchucho, quitaipón o tentemozo. Una primera hipótesis hecha hace tiempo es que estos casos son conservaciones de una tendencia de la lengua antigua: los primeros compuestos de este tipo respondieron al esquema imperativo + régimen o imperativo + vocativo. Luego, el proceso se generalizó con pérdida del valor imperativo, confundiéndose con la tercera persona del singular del presente de indicativo. Sin embargo, en la mayoría de los casos que constituyen este tipo de composición como sacacorchos, quitapenas, etc. no es nada evidente la interpretación del imperativo, sino que parece tratarse más bien del indicativo. Además, las primeras documentaciones que recoge Lloyd nos muestran esquemas que nada tienen que ver con este modelo presidido por el imperativo como secapede, thornasole, mondadientes, etc. ${ }^{62}$. Menéndez Pidal ${ }^{63}$ y Alvar Ezquerra ${ }^{64}$ se inclinan, en general, por reconocer el indicativo, argumentando que es indudable cuando el compuesto equivale una frase adjetiva, por ejemplo, salvamanteles es 'un objeto que se emplea para salvar manteles'.

Lo más admitido en general es que nos encontramos ante un tema verbal, representando por la forma más neutra que puede presentar el verbo: la tercera persona del singular del presente de indicativo, en la que se conserva el sema [+acción] y se pierden las distinciones modales, temporales, aspectuales y personales. De esta manera, los casos que no se adaptan a esta explicación (que son cuantitativamente muy inferiores a los que siguen el modelo regular) pueden ser explicados como un desvío respecto a la norma, bien por la presencia de un imperativo indudable (tentepino), bien por la presencia de cualquier otro tipo de forma (bienmesabe).

Estos casos pueden tener su origen en formaciones expresivas que merecen explicación particular. Por ejemplo, muchos de ellos se pueden explicar en relación con sus referentes, así, un compuesto como tentemozo puede entenderse como una expresión imperativa en que designamos al objeto por la función que queremos que cumpla ${ }^{65}$; otros pueden tener su origen en la lexicalización de un enunciado, como hazmerreír. Recordemos que en América al colibrí se le denominó según la característica que se quería resaltar: si llamaba la atención su

\footnotetext{
${ }^{60}$ Ynduráin, art. cit., pág. 301, Ángel Rosenblat, «El género de los compuestos», Nueva Revista de Filología Española, 7, 1953, págs. 95-112, pág. 103. n. 17 y Lang, op. cit. § 3.3.

${ }^{61}$ Alemany, op. cit., pág. 169 y Bustos Gisbert, op. cit., pág. 258.

${ }^{62}$ Lloyd, op. cit., pág. 17.

${ }^{63}$ Op. cit., pág., 239 n. 1.

${ }^{64}$ Op. cit., pág. 35.

${ }^{65}$ Ibíd.
} 
tamaño, pájaro mosca; pero si impresionaba su capacidad de permanecer suspendido, tentenelaire. Tampoco escaparon a este tipo de composición las situaciones propias de la nueva sociedad que se estaba formando, de ahí que fuese particularmente atinada en algunas denominaciones raciales que se incorporaron sin más al léxico del mestizaje, como ahiteestás (que hace referencia al mantenimiento del color en el cruce racial) o notentiendo (descendiente de progenie sumamente complicada).

Lo más frecuente, como hemos dicho, en este tipo de compuestos es la secuencia de verbo en tercera persona + un complemento, lo que lo convierte en un patrón de gran regularidad. Desde las formaciones más antiguas como alzaparapo, vencemalo, rascaviejas o chupamiel, parece evidente el origen expresivo. También se recurría en el Siglo de Oro a esta composición para la creación expresiva de nombres propios descriptivos de los personajes. Algunos han pasado al acervo común, como trotaconventos.

Esto último no ha sido privativo de la literatura. Pertenece a lo que algunos llaman «genio» de la lengua. El carácter de palabras motivadas en su significado explica su particular frecuencia en el lenguaje afectivo en diversas épocas del idioma, con valores generalmente despectivos (tragaperras, cantamañanas) o humorísticos, y de ahí que también este tipo de composición haya constituido una fuente casi inagotable en la que ha bebido la lengua popular de todos los tiempos para la creación de motes (algunos convertidos en apellidos) tan expresivos como arrastracardos o matamoros.

Por otro lado, frente a otros romances donde este tipo de composición conoce numerosas restricciones, como el rumano ${ }^{66}$, en español, como ha estudiado Lloyd ${ }^{67}$ se añaden designaciones de plantas y, a partir del siglo XII, animales, nombres de profesiones, instrumentos y herramientas, y son numerosos como formaciones esporádicas, sobre todo populares y regionales. Lloyd añade una larga lista de este tipo. Pero son mucho menos numerosas en la norma de la lengua común de España, que prefiere otras formaciones para las mismas designaciones regionales, por ejemplo, sacacorchos frente a descorchador. La época más productiva de este tipo compositivo en español ha sido, quizás, el Siglo de Oro, pues la incorporación de las tierras americanas y las circunstancias concretas del castellano en las diversas zonas incorporó al habla de cada región multitud de estas nuevas formaciones, dado lo regular y expresivo de este patrón. Esto puede explicar porque en la actualidad esta tendencia parece más viva en la norma de muchas regiones americanas que en la de España.

Respecto a sus características, se ha señalado también como un rasgo peculiar de estos compuestos la aparición del morfema de plural en el elemento sus-

\footnotetext{
66 Véase Coseriu, art. cit.

${ }^{67}$ Op. cit., págs. 19 y sigs.
} 
tantivo que acompaña al verbo, siendo el número del compuesto singular (abrelatas). También puede aparecer en singular (agarrapalo), sin que parezca haber razones para tal diferencia, que se ha dado desde las primeras documentaciones que recoge Lloyd. Lo normal es que los componentes se encuentren en unión asindética, sin especificación de la relación gramatical existente entre ellos y que la semántica del compuesto aclara. Con respecto al complemento, la estructura más frecuente es la de verbo + complemento directo (quitamanchas), pero esto no excluye la existencia de abundantes posibilidades distintas (pasacalles, girasol). Otras combinaciones, con adverbio (bogavante, catalejo, mandamás), complemento preposicional (tentempié, saltambanco) o con otro verbo (vaivén), se irán desarrollando progresivamente en la historia del idioma.

En cuanto a las formaciones de verbo + verbo, están caracterizadas por la presencia de dos temas verbales en relación coordinativa o subordinativa, exocéntricos todos semánticamente. Se trata de un grupo marginal de compuestos en castellano (los ejemplos son poco numerosos y se caracterizan por su heterogeneidad que hace difícil en ocasiones cualquier intento de sistematización), de escasa relevancia comparado con los otros grupos.

Se han destacado dos tipos de compuestos en este grupo: 1) ejemplos que parecen responder a un tipo compositivo (lamelame, vaivén); 2) ejemplos que parecen ser el resultado de aglutinaciones sintagmáticas, de lexicalizaciones de sintagmas (correveidile, salsipuedes). Semánticamente (y teniendo en cuenta la relación sintáctica implícita) llama la atención la estructura coordinativa de estos elementos (quitaipón) y las relaciones semánticas que establecen los lexemas componentes, bien por su reiteración intensificadora (picapica), bien por su relación antonímica (moriviví, duermevela, ganapierde, compraventa), bien por pertenecer ambas unidades a un mismo campo semántico (vaivén).

\subsubsection{Compuestos de adverbio + adjetivo o participio}

Ejemplos de este tipo compositivo se encuentran en latín: BENEVOLO, BENEFACIO > benéfico. Los adverbios suelen anteponerse a adjetivos o participios: bienfechor, malfechor, bienaventurado, malaventurado, de los que pueden derivar sustantivos, como bienanventurança. Es un tipo que ha sido desde siempre escasamente productivo en español y se limita a unos pocos adverbios que entran en combinación con adjetivos derivados de verbos (como bien, mal, etc.), pues son participios. Esto plantea el problema de determinar su naturaleza. En el caso de los compuestos de adverbio más participio ¿se trata realmente de compuestos de carácter adjetivo o de compuestos verbales? En estos compuestos los elementos se encuentran en relación adversativa (menospreciado, 'apreciado, pero menos', malaconsejado, 'aconsejado, pero mal'). No es un esquema 
normal en español salvo por gramaticalización (bienquerencia es un derivado de bienquerer, lo que lo incluye en los compuestos de adverbio + verbo). Como observa Alvar Ezquerra ${ }^{68}$, los adverbios que entran en estas formaciones poseen un significado contrario, adversativo y la relación adverbial que se produce en el interior de ellos se refiere a la elección realizada con respecto a la otra posibilidad: alguien «intencionado» puede serlo «bien»o «mal», aunque no siempre exista la pareja de compuestos con bien y mal. Esto explica las escasas formaciones de esta clase en todos los tiempos.

\subsection{La composición verbal}

Son muy escasos los verbos formados por composición, y casi nula la vitalidad de cualquier procedimiento de este tipo. Parece que fue un recurso de composición más propio de la lengua antigua, en la que no debió de conocer gran desarrollo, pues son pocas las formaciones que podemos documentar, sin que apenas se produzcan otras nuevas en épocas posteriores. Sólo existen algunos casos de sustantivo en función de complemento y verbo: pelechar, manlevar; o de adverbio y verbo: ben/maldezir, malferir, menospreciar, menoscabar. Derivados de estos grupos son los casos de adverbio + sustantivo como bienquerencia, malacrianza o bienandanza. Hay ejemplos en los que el carácter compositivo ya no es visible debido a la aglutinación fonética como en FACIEM FERIRE $>$ zaherir. En todos los casos la estructura es siempre de determinante + determinado, que no responde a los modelos actuales en español, por lo que, como hemos dicho, las palabras así compuestas son creaciones viejas en la lengua, a semejanza de las cuales se pueden hacer otras en la lengua actual con rentabilidad baja.

\footnotetext{
${ }^{68}$ Op. cit., pág. 37.
} 
